\title{
Pulmonary Inflammation Induced by a Recombinant Brugia malayi $\gamma$-glutamyl transpeptidase Homolog: Involvement of Humoral Autoimmune Responses
}

\author{
Abdelhila Soussi Gounni, ${ }^{1}$ Katharina Spanel-Borowski, ${ }^{2}$ Miriam Palacios, ${ }^{3}$ \\ Christoph Heusser, ${ }^{4}$ Salvador Moncada ${ }^{3}$, and Edgar Lobos ${ }^{3}$ \\ ${ }^{1}$ Meakins Christie Laboratories, McGill University, Montreal, Canada \\ ${ }^{2}$ Institute of Anatomy, University of Leipzig, Leipzig, Germany \\ ${ }^{3}$ The Wolfson Institute for Biomedical Research, University College London, London, England \\ ${ }^{4}$ Novartis Pharma AG, Transplantation Immunology, Basel, Switzerland \\ Contributed by L. Miller. Accepted February 20, 2001
}

\begin{abstract}
Background: A major allergen from the lymphatic filarial parasite Brugia malayi implicated in the pathogenesis of tropical pulmonary eosinophilia (TPE) has recently been cloned and identified as the homolog of the membrane-bound mammalian enzyme $\gamma$-glutamyl transpeptidase $(\gamma-\mathrm{GT})$. Patients with acute TPE show autoreactive antibodies against endogenous $\gamma$-GT from the pulmonary epithelium.

Materials and Methods: Recombinant B. malayi $\gamma-\mathrm{GT}$, alone or adsorbed to aluminium hydroxide (AL), was used in a BALB/C mouse model to analyze its antigenic/allergenic potential, its potential to induce pulmonary inflammation, and its capacity to induce autoreacting antibodies.

Results: Mice immunized with $B$. malayi $\gamma$-GT showed significant levels of $\gamma$-GT-specific IgG1, IgG2a, IgG3, IgA, IgE antibodies, and mild blood eosinophilia, even in the absence of adjuvant. Intranasal challenge with $B$. malayi $\gamma$-GT induced peribronchial and perivascular inflammation
\end{abstract}

characterized by a mixed infiltrate of lymphocytes, neutrophils, eosinophils, and macrophages. Both IL-4 and IFN- $\gamma$ were detected in the peripheral blood and in the bronchoalveolar lavage fluid of immunized and intranasally challenged mice. Histological analysis of murine lungs using affinity-purified antibodies from mice immunized with the parasite's $\gamma$-GT revealed the presence of autoimmune antibodies against pulmonary epithelium. Western blot analysis identified the $55 \mathrm{kDa}$ heavy chain subunit of the murine $\gamma$-GT as the target of autoreactive / crossreacting antibodies. Conclusion: Our data from the in vivo mouse model demonstrate the potent allergenicity/antigenicity of $B$. malayi $\gamma$-GT, and its capacity to induce pulmonary inflammation upon intranasal challenge. This leads to breakdown of tolerance against endogenous murine $\gamma$-GT. Thus, humoral autoimmunity against the airways epithelium may contribute to the pathogenesis of TPE.

\section{Introduction}

Pulmonary hypersensitivity reactions to microfilarial antigens from the lymphatic filarial parasites Wuchereria bancrofti or Brugia malayi, as well as high blood and pulmonary eosinophilia, is an initial hallmark of acute tropical pulmonary eosinophilia syndrome (TPE). Individuals with TPE show elevations of anti-filarial antibodies of all classes; in particular, circulating IgE antibodies are extremely high (1). The clinical features of TPE include nocturnal asthma, cough, wheezing, general malaise, and lung function changes characteristic of an inflammatory interstitial lung disorder (2).

The absence of circulating microfilariae (Mf) from the blood of patients with TPE is considered the result of their clearance in the lungs as demonstrated by histopathological studies of lung biopsies

Address correspondence and reprint requests to Dr. Edgar Lobos, University of Leipzig, Institute of Anatomy Liebigstrasse 13, 04103 Leipzig, Germany Phone: +49-341-9722051. Fax: +49-3419722009 E-mail address: lobe@medizin.uni-Leipzig.de revealing trapped, often degenerating, Mf $(3,4)$. The accumulation of high levels of filarial-specific antibodies detected in the bronchoalveolar lavage fluid (BAL) of patients with acute TPE has been proposed to mediate clearance of $\mathrm{Mf}$ in the pulmonary vasculature, and probably contributes to the pathology of TPE. The restricted recognition of only a small number of filarial antigens by pulmonary IgG and IgE antibodies would argue in favor for their involvement in the pathogenesis of TPE (5).

We previously characterised Bm23-25, a major IgE-inducing filarial antigen from Brugia malayi, as an important target of humoral and cellular immune responses in the lungs of patients infected with W. bancrofti exhibiting acute TPE (6). Bm23-25 is expressed not only by adult worms but also in the microfilarial stage and is able to induce IgE synthesis in vitro from peripheral blood mononuclear cells of patients with TPE or other filarial symptoms. The Bm23-25 filarial allergen, although involved in strong humoral and cellular responses, was present in soluble protein extract in amounts below the 
detection range for either protein sequencing or the raising of specific antibodies (6).

Immunoscreening of $B$. malayi cDNA libraries with affinity-purified Bm23-25-specific IgE antibodies from patients with acute TPE identified a corresponding cDNA. We have established using sequence analysis that the identified parasite gene encodes a protein that is homolog to mammalian $\gamma$-glutamy 1 transpeptidase ( $\gamma$-GT) (7). $\gamma$-GT is a key multifunctional enzyme involved in the salvage of glutathione (GSH) and in cellular processes, depending on the oxidation/reduction of GSH (8).

We have shown that filarial B. malayi $\gamma-\mathrm{GT}$ is able to trigger autoimmunity against human $\gamma$-GT present in lung epithelial cells (7). Therefore, breakdown of tolerance toward the human airways epithelium induced by $\gamma$-GT from filarial parasites may play a hitherto unknown role in the pathogenesis of microfilariae-induced tropical pulmonary eosinophilia. Murine models of pulmonary inflammation established using live Mf and/or total protein extracts from Mf exhibit several features of human TPE and have given valuable insights into the cytokine network involved in the murine pulmonary inflammation (9-11). However, structural identification of parasite antigens directly involved in the pathogenesis of this syndrome and of their pathophysiological mechanisms are lacking. The intrinsic capacity of nematodes to induce Th2 responses suggests the presence of defined parasitederived molecules capable of up-regulating early production of IL-4 by several cell types.

In this study, we analyzed the potential of the purified recombinant $B$. malayi $\gamma$-GT to mediate pulmonary inflammation in vivo using a BALB/c mouse model, and we determined whether immunization with filarial $\gamma$-GT would induce antibodies reactive with the mouse's pulmonary epithelium. The data show that immunization with filarial $\gamma$-GT, aqueous or adsorbed to aluminium hydroxide, induced high levels of $\gamma$-GT-specific antibodies, and nonpolarized Th responses. Airways histology performed $72 \mathrm{hr}$ after the intranasal challenge with filarial $\gamma$-GT revealed the presence of massive pulmonary inflammation with significant perivascular, peribronchial, and alveolar cellular infiltration. We demonstrate that autoantibodies induced by immunization with parasite's $\gamma$-GT react with the murine $\gamma$-GT present on the pulmonary epithelium. These $\gamma$-GT-specific autoantibodies probably contribute to the local pulmonary pathology observed in the TPE syndrome.

\section{Materials and Methods}

\section{Antibodies and Reagents}

Unlabeled goat anti-mouse IgG was purchased from Sigma (Sigma Chemical Co, Poole, UK). Mouse immunoglobulins standards (IgA, IgGl, IgG2a, and IgG3), and biotin-conjugated goat anti-mouse $\operatorname{IgA}$, IgG1, IgG2a, and IgG3 $(\alpha, \gamma 1, \gamma 2 \mathrm{a}, \gamma 3$ heavy chain- specific) were purchased from Southern Biotechnology Associates, Birmingham, AL. Gamma bind plus Sepharose was from Pharmacia (Pharmacia Fine Chemicals, Piscataway, NY). Rat anti-mouse IgE Mab4B3-39, mAb, and IgE standards were from Dr. Christoph Heusser (Novartis Inc, Basel, Switzerland). Extravidin phosphatase alkaline was obtained from GIBCO-BRL (Bethesda, MD), rat antimurine IFN- $\gamma$, and rat anti-murine IL-4 Abs were from Endogen (Minikit, Bioreba, Switzerland).

\section{Antigen Preparation}

Recombinant B. malayi $\gamma$-GT was prepared as described previously (7). Briefly, the pJC20- $\gamma$-GT.1 plasmid was expressed in the protease-deficient $E s$ cherichia coli BL21(DE3). Cells were grown in LuriaBertani broth supplemented with $50 \mu \mathrm{g} / \mathrm{ml}$ ampicillin and $0.2 \%$ glucose $(\mathrm{w} / \mathrm{v})$ until $\mathrm{OD}_{600}=0.6$; protein synthesis was induced by addition of isopropyl $\beta$-D thiogalactoside to a final concentration of $0.5 \mathrm{mM}$, and centrifuged $3 \mathrm{hr}$ later at $4000 \times \mathrm{g}$ for $10 \mathrm{~min}$. After washing with $0.9 \% \mathrm{NaCl}$, cells were resuspended in lysis buffer A $(6 \mathrm{M} \mathrm{Gu} \mathrm{HCl}, 0.1 \mathrm{M}$ Na-phosphate, $0.01 \mathrm{M}$ Tris-HCl, $\mathrm{pH}$ 8.0) for $1 \mathrm{hr}$ at room temperature. The lysates were centrifuged at $50,000 \times \mathrm{g}$ for $\mathrm{lh}$ at $4^{\circ} \mathrm{C}$. The supernatants containing the soluble recombinant filarial $\gamma$-GT were incubated with $50 \%$ Ni-NTA agarose for $3 \mathrm{hr}$ at room temperature. The recombinant filarial allergens were washed and eluted according to a standard protocol (QIAGEN Express system, Qiagen). The collected fractions were analysed by SDS-PAGE, fractions containing pure $\gamma$-GT were pooled and stepwise dialyzed for $72 \mathrm{hr}$ first with modified buffer C (3M urea, 0.1M Na phosphate, $0.01 \mathrm{M}$ Tris- $\mathrm{HCl}, \mathrm{pH}$ 6.3) and then with PBS. The pooled fractions were passed over a polymycin affinity column (Detoxi-gel column $1 \mathrm{ml}$ bed volume; Pierce Chemical Co., Rockford, IL) followed by determination of the protein concentration using bovine serum albumin (BSA) as a standard and the BCA protein assay reagent (Pierce Chemical Co., Rockford, IL). The antigen solution was tested for the presence of LPS by its capacity to up-regulate iNOS in cultures of murine bone marrow macrophages isolated as described (12). No up-regulation of iNOS or of its end product nitrate was observed after addition of the recombinant filarial antigen solution (data not shown).

\section{Animals}

Specific pathogen-free BALB/c female mice (6-8 weeks old) were obtained from IFACREDO (Lyon, France) and maintained under standard pathogenfree conditions.

\section{Sensitization and Antigen Challenge Protocol}

Dose titration experiments were carried out to determine the optimal amounts of recombinant $B$. malayi $\gamma$-GT inducing maximal $\gamma$-GT-specific immune responses. In brief, naive Balb/c mice (six mice/group 
for the determination of IgG1, IgG2a, and IgG3 or four mice/per group for IgA, IgE and total IgE) were immunized with $10 \mu \mathrm{g}$ of recombinant filarial $\gamma$-GT, either aqueous $(\gamma$-GT) or pre-absorbed to $4 \mathrm{mg}$ of aluminium hydroxide ( $\gamma$-GT/AL), i.p. on days 0,15 , and 28 day followed by i.v. immunization on days 35 and 61. In addition, a control group of six mice were immunized with $10 \mu \mathrm{g}$ of $B$. malayi microfilarial protein extract using the same antigen challenge protocol (data not shown). Antibody production was determined in sera of mice collected prior to the antigen challenge. Control mice were injected with $0.9 \%$ sodium salt in a similar immunization schedule. Mice were bled from the tail veins and serum stored at $-80^{\circ} \mathrm{C}$ until assayed for antibody titres.

\section{Bronchoalveolar Lavage}

On day 72 of the experiment, mice were anaesthetized with isofluorane and challenged by the intranasal route with $10 \mu \mathrm{g}$ of $\gamma$-GT alone, $\gamma$-GT/AL, or saline in a volume of $50 \mu \mathrm{l}$. Seventy-two hr after the airways challenge, mice were anaesthetized with a lethal dose of urethane, the trachea canulated, and bronchioalveolar lavage (BAL) performed by five washes with $0.3 \mathrm{ml}$ saline injected into the lungs via the trachea. Cells in the BAL fluid were centrifuged $\left(10 \mathrm{~min}, 4^{\circ} \mathrm{C}\right.$, $700 \mathrm{~g}$ ), and supernatant collected and stored at $-80^{\circ} \mathrm{C}$ for analysis of cytokine content. After resuspension in PBS, total cells were counted in a haemocytometer (Coulter Counter, Hertfordshire, UK). Differential cell counts were performed on cytospin preparations stained with May-Gruenwald-Giemsa by classification of 300 cells on standard hematological criteria.

Analysis of B. malayi $\gamma$-GT-specific IgG1, IgG2a, and IgG3 Subclasses

Maxisorb microtitre plates were coated with 0.5 $\mu \mathrm{g} / \mathrm{ml}$ recombinant $B$. malayi $\gamma-\mathrm{GT}$ in carbonate buffer $\mathrm{pH} 9.6$ overnight at $4^{\circ} \mathrm{C}$. The concentration of $\gamma$-GT-specific IgG subclasses was estimated by comparison to a standard curve of high-titre serum pool with known concentration of $\gamma$-GT subclass antibodies over a range of dilutions (1/20-1/12800). Plates were incubated overnight at $4^{\circ} \mathrm{C}$ with serially diluted sample sera $(1: 100,1: 300,1: 900)$ in duplicate. After washing, plates were incubated for $1 \mathrm{hr}$ with biotinylated goat anti-mouse IgGl, IgG2a, or IgG3 (1:1000). After a further wash, plates were incubated for $\mathrm{lh}$ at $37^{\circ} \mathrm{C}$ with extrAvidin phosphatase alkaline (1:1000). Finally, plates were developed with p-nitro-phenyl phosphate disodium $1 \mathrm{mg} / \mathrm{ml}$ (Sigma Chemical Co., St. Louis, MO). The absorbance at $405 \mathrm{~nm}$ was determined using a microplate reader. Sera from preimmune mice and from mice immunized with physiologic sodium salt alone were used as negative controls in each experiment.

\section{Quantitation of Total IgE}

Previous dose-response experiments showed a direct correlation between the induced amounts of total/ specific IgE and the amount of filarial $\gamma$-GT used for immunization in a concentration range of $100 \mathrm{ng}$ to $30 \mu \mathrm{g}$ (not shown). Sera were collected and stored at $-80^{\circ} \mathrm{C}$ until required. Blocking IgG antibodies were removed by preadsorption at $4^{\circ} \mathrm{C}$ to protein $\mathrm{G}$ Sepharose (Pharmacia, Piscataway, NY) overnight at $4^{\circ} \mathrm{C}$ as previously described (6). Levels of total mouse IgE were detected by ELISA, as described (13). In brief, Maxisorp plates (Nunc) were coated with mAb $4 \mathrm{~B} 3-39$ rat anti-mouse $\operatorname{IgE}(20 \mu \mathrm{g} / \mathrm{ml})$ for $2 \mathrm{hr}$ at $37^{\circ} \mathrm{C}$ followed by overnight incubation at $4^{\circ} \mathrm{C}$. Plates were washed with PBS pH 7.2 and then saturated with $\mathrm{PBS} / 1 \% \mathrm{BSA}(\mathrm{w} / \mathrm{v})$ for $1 \mathrm{hr}$ at $37^{\circ} \mathrm{C}$. On each plate, serial dilutions of serum samples (1:20, 1:50, 1:100) and the appropriate known amounts of mouse IgE to construct a standard curve were incubated for $2 \mathrm{hr}$ at $37^{\circ} \mathrm{C}$. After washing with PBS-Tween, biotinylated rat anti-mouse $\operatorname{IgE}(1: 5000)$ was added to detect bound $\operatorname{IgE}$ for $1 \mathrm{hr}$ at $37^{\circ} \mathrm{C}$, followed by a further washing step and incubation with extrAvidin alkaline phosphatase $(1: 1000)$ for $1 \mathrm{hr}$ at $37^{\circ} \mathrm{C}$. The amount of bound antibody was revealed by incubating each well with $150 \mu \mathrm{l}$ of $1 \mathrm{mg} / \mathrm{ml}$ p-nitrophenylphosphate in carbonate buffer ( $\mathrm{pH}$ 9.6). The absorbance was determined as described above. Sera from mice immunized with physiologic sodium salt alone were used as negative controls in each experiment.

\section{Analysis of B. malayi $\gamma$-GT-specific IgE}

Maxisorb plates were coated with recombinant filarial $\gamma$-GT $(5 \mu \mathrm{g} / \mathrm{ml})$ in carbonate buffer $\mathrm{pH} 9.6$ for $2 \mathrm{hr}$ at $37^{\circ} \mathrm{C}$, followed by overnight incubation at $4^{\circ} \mathrm{C}$. On each plate, a standard curve of a high-titre standard serum pool with known concentrations of $\gamma$-GTspecific IgE was included. Plates were saturated and incubated with serially diluted IgG pre-adsorbed sera (1:20, 1:50, 1:100) overnight at $4^{\circ} \mathrm{C}$ and processed as described above.

\section{Analysis of B. malayi $\gamma$-GT-specific IgA}

Plates were coated with recombinant B. malayi $\gamma$-GT $(5 \mu \mathrm{g} / \mathrm{ml})$ as previously described. A standard serum pool with known amounts of $B$. malayi $\gamma$-GT-specific IgA was run on each plate. Following the saturation step and addition of diluted serum samples (1:50, $1: 100,1: 500)$, plates were incubated overnight at $4^{\circ} \mathrm{C}$. The same conditions as for the detection of $\operatorname{IgE}$ Abs were used to develop the plates.

\section{Cytokine Analysis}

The presence of IFN- $\gamma$ and IL- 4 in the sera of mice immunized with recombinant $B$. malayi $\gamma-\mathrm{GT}$ or in the BAL from sensitized and antigen-challenged mice was measured using commercial kits with monoclonal rat anti-murine IFN- $\gamma$ (Dynatech, Embrach, Switzerland) or rat anti-murine IL-4-both according to the manufacturer's instructions (Minikit, Endogen). After overnight incubation with the diluted samples and standards, bound cytokines were detected after washing by sequential incubation 
with the respective biotinylated anti-murine IFN- $\gamma$ or anti-IL-4 Ab, followed by incubation with horseradish peroxidase-conjugated streptavidin (1:2000). Plates were incubated for $3 \mathrm{hr}$ with the conjugates and, after washing, with the substrate 1,1'-trimethylenebis 4-formylpyridinium bromide (TMB). The plates were read on an ELISA reader at $620 \mathrm{~nm}$. The sensitivity of the IL- 4 and IFN $-\gamma$ ELISA was $<5 \mathrm{pg} / \mathrm{ml}$ and $<15 \mathrm{pg} / \mathrm{ml}$ respectively.

\section{Blood Eosinophilia}

Mice were bled from the tail vein on days $0,15,21$, $35,42,61$, and 65 prior to the respective immunization. Blood smears were stained with Diff-Quick stain (Merz \& Dade AG, Düdingen, Switzerland), and eosinophils were quantified by differential cell counts from at least 300 leukocytes using standard hematological criteria. The fold increase in peripheral blood eosinophils represents the ratio of the mean value between the eosinophil number at each given time point and the baseline eosinophil number on day 0 .

\section{Histology}

The lungs were removed, fixed in $4 \%$ phosphatebuffered formalin, embedded into paraffin, and sectioned. Sections $(10 \mu \mathrm{m})$ were stained either with hematoxylin or with Sudan Red for demonstration of eosinophil granules as previously described (14). For assessment of the airways inflammation induced by the intranasal challenge with recombinant filarial $\gamma$-GT, lung sections were screened and pictures taken with a light microscope (Axiovert 2, Zeiss, Jena).

\section{Immunohistochemistry}

Lung sections from untreated BALB/c mice were mounted on object slides previously coated with 3-aminopropyltrietoxysilane (Sigma Chemicals, St. Louis, MO). Indirect immunolabeling detection of autoantibodies against the murine $\gamma$-GT was carried out after leaving sections in standard phosphate saline buffer (PBS) for $24 \mathrm{hr}$ at $4^{\circ} \mathrm{C}$. Incubation in $0.3 \% \mathrm{H}_{2} \mathrm{O}_{2}$ in absolute methanol for 5 min was carried out to quench endogenous peroxidase activity followed by washes in PBS $(2 \times 10 \mathrm{~min})$ and in $0.05 \mathrm{M}$ TBS (Tris-HCl pH 7.6). Unspecific antibody binding was blocked by 30-min incubation with TBS containing $2.5 \%$ normal goat serum (DAKO, Hamburg, Germany). Immunolabeling was carried out with the avidin-biotin-horseradish peroxidase complex technique (Vectastain Elite ABC kit, Vector Laboratories, Alexis, Gruenberg, Germany). Sections were incubated overnight at $4^{\circ} \mathrm{C}$ with murine affinity purified antibodies against the $\gamma$-GT $B$. malayi diluted 1: 1000 in $2.5 \%$ PBS followed by incubation with biotinylated goat anti-mouse IgG, Vector BA-9200 (1: 200). They were then incubated with the ABC complex, and peroxidase activity was detected histochemically with the 3,3, diamino benzidine tetrahydrochloride dihydrate substrate (Aldrich, Basel, Switzerland). The sections were then mounted on Canada Balsam (15). Pre-immune mouse serum was used as a control.

\section{Affinity Purification of Anti- $\gamma$-GT Antibodies}

Purified recombinant $B$. malayi $\gamma$-GT antigen separated previously by SDS-PAGE was electrophoretically transferred to nitrocellulose. The nitrocellulose strips were incubated overnight with a serum pool from mice immunized against the filarial $\gamma$-GT homolog (1:100), and the antibody was eluted as described (6).

\section{Murine $\gamma$-GT Activity Staining in Native Gels}

In order to enrich pulmonary $\gamma$-GT, in situ detection of its enzymatic activity, followed by isolation of the protein from the stained area, was performed. Murine lungs were dissected, washed in cold PBS, and extracted on ice in homogenization buffer (20 mM HEPES, $0.2 \mathrm{M}$ sucrose, pH 7.2, ImM EDTA, soybean trypsin inhibitor $[20 \mu \mathrm{g} / \mathrm{ml}]$, leupeptin [20 $\mu \mathrm{g} / \mathrm{ml}]$, pepstatin [5 $\mu \mathrm{g} / \mathrm{ml}]$, E-64 [5 $\mu \mathrm{g} / \mathrm{ml}]$, bestatin $[5 \mu \mathrm{g} / \mathrm{ml}]$, aprotinin $[5 \mu \mathrm{g} / \mathrm{ml}] \quad 0.1 \mathrm{mM}$ PMFS). Lung proteins were centrifuged at $10,000 \times \mathrm{g}$ for $10 \mathrm{~min}$ at $4^{\circ} \mathrm{C}$. Samples containing $150 \mu \mathrm{g}$ protein were incubated with Triton X-100 to a final concentration of $0.5 \%$ on ice for $30 \mathrm{~min}$. Sample buffer was added and probes loaded into a $6 \%$ nondenaturing Ornstein-Davis gel and run at $20 \mathrm{~mA}$ for $4 \mathrm{hr}$. Staining for $\gamma$-GT activity was carried out as described (16). Briefly, after electrophoresis gels were equilibrated in $100 \mathrm{mM}$ Tris $\mathrm{HCl} \mathrm{pH} 7.4,16 \mathrm{mM}$ $(\mathrm{Gly})_{2}$. After $20 \mathrm{~min}$, this was replaced by buffer containing $200 \mu \mathrm{g} / \mathrm{ml}$ gamma-glutamyl-4-methoxy-2naphthylamide (GMNA), and $400 \mu \mathrm{g} / \mathrm{ml}$ Fast Blue RR. After 20-30 min incubation at room temperature, the solution was replaced by $100 \mathrm{mM} \mathrm{CuSO}_{4}$.

\section{Western Blot Analysis of Isolated Murine $\gamma-G T$}

After in situ determination of $\gamma$-GT activity, the broad, dark-orange band was cut and protein isolated as previously described (17). The isolated murine $\gamma$-GT was submitted to $12.5 \%$ SDS-PAGE and transferred electrophoretically to nitrocellulose. The presence of autoimmune antibodies against mouse $\gamma$-GT was identified using serum from mice immunized with B. malayi $\gamma$-GT (1:1000). Primary antibodies were localized with alkaline phosphatase-conjugated goat anti-mouse IgG (1:3000). Alkaline phosphatase activity was localized using nitro blue tetrazolium bromochloroindolyl phosphate substrate (Kirkegaard \& Perry Laboratories).

\section{Statistical Analysis}

Differences between groups were evaluated for statistical significance using the method of KruskallWallis and the Mann-Whitney test for nonparametrical data. Differences with $p<0.05$ were determined to be statistically significant. 


\section{Results}

\section{Kinetics and IgG Antibody Subclasses Induced by B. malayi $\gamma-G T$ in $B A L B / c$ Mice}

We analyzed the Igs antibody response in mice sensitized either to aqueous or aluminium-precipitated recombinant filarial $\gamma$-GT by ELISA. The predominant IgG antibody subclass induced during the 65 days of our immunization scheme by $B$. malayi $\gamma$-GT was IgGl (Figure 1, A). Induction of $\gamma$-GTspecific IgGl antibody levels occurred early as significant levels were detectable already by day 15 after the primary challenge. IgGl was the predominant subclass until day 35 before the first i.v. Ag challenge. Two weeks after the second i.p. injection (day 28), significantly elevated concentrations of $\gamma$-GT-specific IgGl and IgG2a antibodies were detected in both groups of immunized mice (Figure 1, A and B, respectively). The synthesis of filarial $\gamma$-GT-specific IgGl and IgG3 antibodies was greatly enhanced after the first i.v. antigen challenge as measured on day 42; these levels decreased in the subsequent weeks and rose again after the second i.v. Ag challenge as measured on day 65 (Figure 1, A and C). $\gamma$-GT-specific IgG2a and IgG3 antibodies were enhanced after the first i.v. boost but not after the secondary (Figure 1, B and C, respectively). Antibody concentrations induced by alum-absorbed antigen $(\gamma$-GT/AL $)$ were more pronounced than those induced after immunization with $\gamma$-GT alone, but the difference did not reach statistical significance $(p>0.05)$. No $\gamma$-GT-specific antibodies were detected in serum from animals immunized with vehicle alone (data not shown).
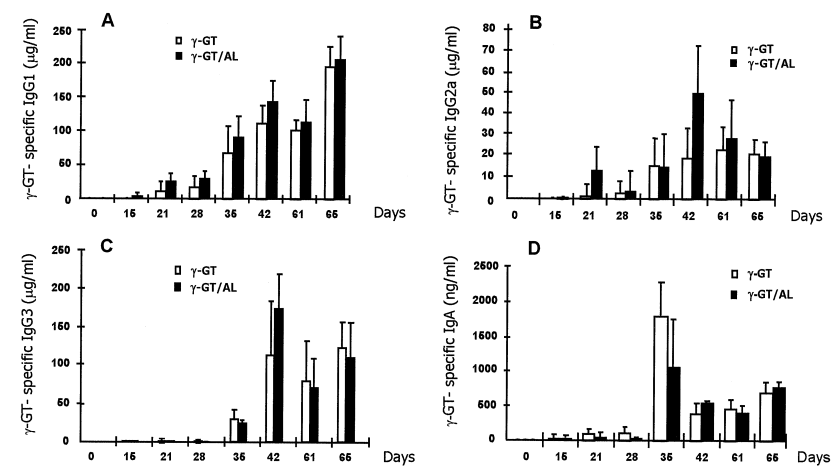

Fig. 1. Time course and intensity of the $B$. malayi $\gamma$-GT-specific antibody isotype responses in the sera of BALB/c mice immunized with aqueous or Alumprecipitated $B$. malayi $\gamma$-GT. Mice $(\mathrm{n}=6)$ from each group were injected i.p. with $10 \mu \mathrm{g}$ recombinant $\gamma$-GT or $\gamma$-GT/AL on days 0,15 , and 28 and i.v. on days 35 and 61. Individual sera obtained prior to the immunizations were tested for the presence of $\gamma$-GT-specific antibodies by class- and subclass-specific ELISA. Bars represent the geometric mean value \pm SD of 6 individuals, $\gamma$-GT-specific Igs' concentrations are either in $\mu \mathrm{g} / \mathrm{ml}$ or $\mathrm{ng} / \mathrm{ml}$. Figure 1A, IgG1; Figure 1B IgG2a; Figure 1C. IgG3; Figure $1 D$. IgA. Similar results were observed in 3 repeated experiments. They all were significantly different from the saline-immunized group since no specific antibodies were detected in this group.
Kinetics and Levels of $\gamma$-GT-specific IgA

Strikingly, immunization with recombinant $B$. malayi $\gamma$-GT stimulated the production of soluble IgA antibodies, which include the Ig isotype produced at mucosal surfaces. In contrast to the other isotype antibodies, $\gamma$-GT-specific IgA levels were greatly enhanced (16-fold and 34-fold, respectively) only after the third i.p. immunization on day 28 (Figure 1D). Interestingly, prior to the first i.v. challenge on day 28, the production of IgA was significantly higher in the mice immunized with $\gamma$-GT alone than in the group of mice immunized with adjuvant $(p=0.043)$.

\section{Induction of Total IgE}

Acute TPE correlates with extremely elevated levels of total and filarial-specific IgE antibodies in the serum and lung epithelial lining fluid $(1,2,5)$. Therefore, we investigated the potential of the recombinant parasite $\gamma$-GT either alone or in the presence of the adjuvant Alum to enhance the synthesis of total IgE antibodies in our BALB/c animal model. Total IgE levels measured by ELISA are shown in Figure 2A. $\gamma$-GT/AL-immunized mice showed earlier significant increases of total $\operatorname{IgE}$ antibody levels that were already detectable on days 21 and 28, as seen in Figure 2A ( $p=0.03$ for both time points), when compared to saline-immunized animals. The second i.v. immunization led to an additional significant increase on day 65 in total $\operatorname{IgE}$ in both $\gamma$-GT-immunized groups $(p=0.34)$. The second i.v. challenge markedly up-regulated the synthesis of total IgE (15-fold increase in $\gamma$-GT/ALimmunized mice and 8 -fold in $\gamma$-GT administered without adjuvant when compared to baseline data on day 0 ).

\section{Induction of $\gamma$-GT-specific IgE}

Because cross-linking of parasite allergen-specific IgE and allergen on the surface of effectors cells is

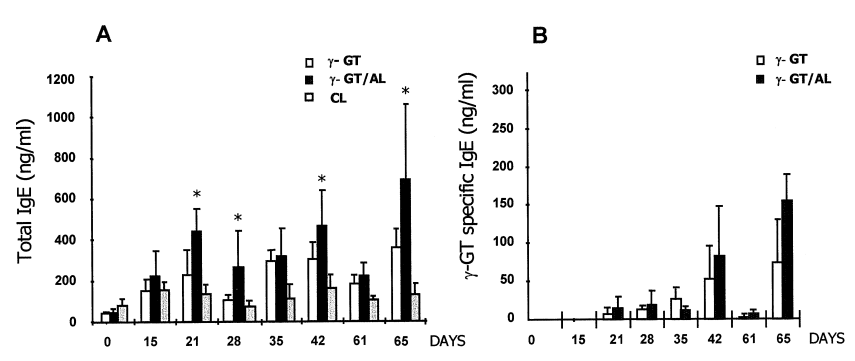

Fig. 2. Time course and intensity of serum total IgE (A), and $B$. malayi $\gamma$-GT-specific IgE (B) antibody responses to recombinant filarial in $\gamma$-GT-immunized mice. Sera from mice $(n=4)$ were pre-adsorbed overnight to protein $G$ sepharose to remove blocking IgG before the measurement of total IgE and $\gamma$-GT-specific IgE by ELISA as described in Materials and Methods. Values are expressed as geometric mean $\pm \mathrm{SD}, \gamma$-GTchallenged mice, $\gamma$-GT/AL, or saline-challenged. * $p=0.021$ significantly different values between $\gamma$-GT, and $p=0.034$ between $\gamma$-GT/AL and control group (CL), respectively. 
directly involved in the immediate hypersensitivity reactions observed in patients with acute TPE, we investigated the capacity of the soluble recombinant filarial $\gamma$-GT to induce antigen-specific IgE antibodies in our mouse model. One week after the second i.p. challenge (day 21), $\gamma$-GT-specific IgE antibodies were already detected in serum collected from both groups of immunized mice (Figure 2B). Immunization by the i.v. route resulted in a significant induction of $\gamma$-GT-specific IgE levels in both groups of immunized mice. A 7- and a 2-fold increase in $\gamma$-GT/AL- and $\gamma$-GT-immunized mice was detected when compared to values measured after the i.p. immunization on day 35. Remarkably, the second i.v. immunization stimulated an 18 -fold and a 9-fold increase in $\gamma$-GT-specific IgE in both groups respectively. $\gamma$-GT-specific IgE antibodies represented approximately $7 \%$ of the total IgE on day 28 after the Ag immunization with Alum with further dramatic increases to approximately $17 \%$ and $20 \%$ after the first and second i.v. immunization, respectively. A similar increase in the percentage of specific IgE to total IgE was observed in the group immunized with $\gamma$-GT alone as seen Figure 2B. Therefore these data from the animal model clearly confirm our previous findings that the filarial antigen $\gamma$-GT induces high levels of specific IgE in vitro.

\section{Time Course of Peripheral Blood Eosinophilia}

To evaluate the changes in peripheral blood eosinophils during the course of immunization with $\gamma$-GT, numbers of peripheral blood eosinophils were determined in $\gamma$-GT, $\gamma$-GT/AL, and control groups were counted at days $0,15,28,35,61$, and 65 and compared to the baseline data obtained at day 0 . As shown in Figure 3, blood eosinophil numbers were increased on day 21 after the second i.p. boost with $\gamma$-GT immunized mice showing a 4 -fold and

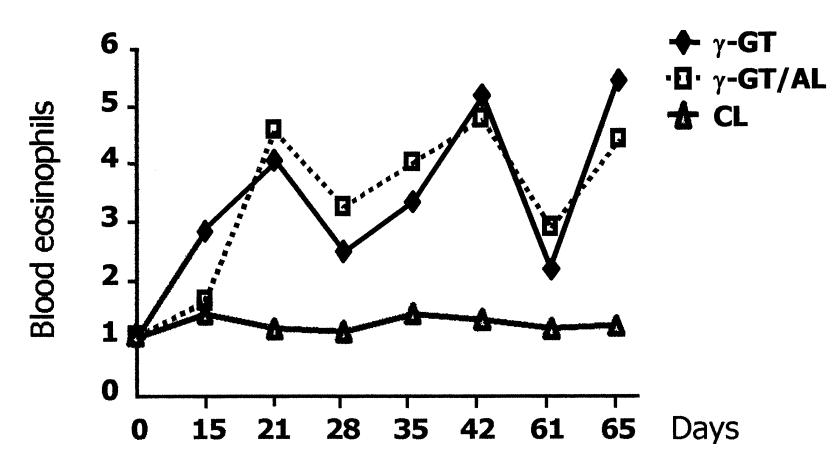

Fig. 3. Time course and intensity of the blood eosinophilia induced by the immunization of BALB/c mice with aqueous $B$. malayi $\gamma$-GT (closed diamonds), $\gamma$-GT/AL (open squares), and vehicle (closed triangles). Data are presented as X-fold increase given by the ratio of the mean value of total eosinophils at each time point and the baseline eosinophil numbers as measured on day $0(n=6$ mice per group $)$.
$\gamma$-GT/AL showing a 4.5-fold increase in eosinophils. Additional i.p. and i.v. immunizations did not increase the numbers of eosinophils in the peripheral blood. Mice from the control group showed no significant increase in peripheral blood eosinophilia.

Serum Cytokine Profile After i.p. Immunization with B. malayi $\gamma-G T$

Analysis of the cytokine pattern of pooled serum from mice taken 15 days after the primary immunization indicated the presence of significantly elevated concentrations of both IL-4 and IFN- $\gamma$ in the blood of immunized mice (Figure 4A). A significant 6-fold up-regulation in the levels of IL-4 was observed after the first i.p. immunization with either $\gamma$-GT with alum or $\gamma$-GT in aqueous solution when compared to the basal levels of this cytokine. IFN- $\gamma$ levels were also significantly enhanced (17-fold) in mice immunized in the presence of adjuvant, which contrasts the 8-fold increase observed in the group immunized with the parasite's antigen alone (Figure 4B).

\section{Airway Inflammation in Mice Intranasally Challenged with $\gamma-G T$}

Intranasal $\gamma$-GT provocation of immunized, but not control, mice resulted in a selective pulmonary infiltration of inflammatory cells. Table 1 shows the total cell and differential counts from BAL $72 \mathrm{~h}$ after the intranasal challenge, showing pronounced increases of neutrophils, lymphocytes, and eosinophils. The absolute numbers of inflammatory cells were similarly elevated in both groups of $\gamma$-GT-immunized mice and were significantly higher than in control animals $(p<0.05)$. With the exception of monocytes/ macrophages numbers, which were actually lower in the $\gamma$-GT/AL-immunized animals, all leukocyte types were significantly higher
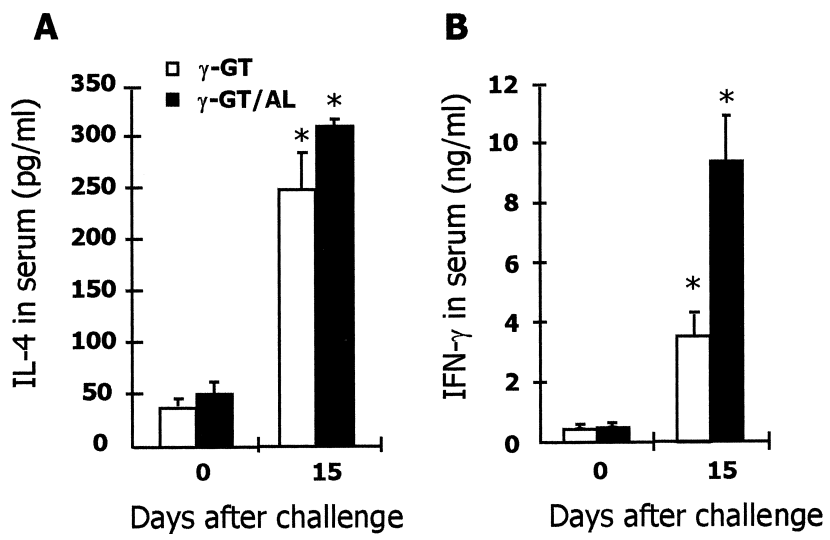

Fig. 4. Levels of IL-4 (A) and IFN- $\gamma(B)$ induced by $B$. malayi $\gamma$-GT in the serum from BALB/c mice 15 days after the primary immunization with $\gamma$-GT, $\gamma$-GT/AL or vehicle. Data represent the mean $\pm \mathrm{SD}(\mathrm{n}=4) . \mathrm{P}=0.021$, * significant differences between each group of $\gamma$-GT-immunized mice and the control group 9 (Day 0). 
Table 1. Cellular content in BAL fluid of mice immunized with B. malayi $\gamma$-GT alone or with Alu $(\gamma$-GT/AL) or saline*

\begin{tabular}{lccccc}
\hline Treatment & $\begin{array}{c}\text { Total Cells } \\
\left(\times \mathbf{1 0}^{3} / \mathbf{m l}\right)\end{array}$ & Mono/Macro & Lymph & Neu & Eos \\
\hline Saline & $350 \pm 56$ & $320 \pm 39$ & $8.4 \pm 0.5$ & $27 \pm 1.1$ & $1.7 \pm 0.2$ \\
$\gamma$-GT & $1260 \pm 115$ & $323 \pm 31$ & $185 \pm 57$ & $523 \pm 71$ & $14.6 \pm 10$ \\
$\gamma$-GT/AL & $730 \pm 106$ & $180 \pm 54$ & $205 \pm 12.3$ & $294 \pm 11$ & $13.8 \pm 3.8$ \\
\hline
\end{tabular}

*Values represent geometric mean \pm SD $(n=4)$. As indicated in Materials and Methods, intranasal challenge was carried out 71 days after immunization. BAL was performed $72 \mathrm{~h}$ after the intranasal challenge.

in $\gamma$-GT-immunized mice than in control animals $(p<0.05)$.

Histological analysis of the lungs of salinetreated mice showed no pathology (Figure 5, A and B). Lungs of $\gamma$-GT-challenged mice $72 \mathrm{hr}$ after the intranasal challenge confirmed the previous findings of the development of peribronchial and perivascu-

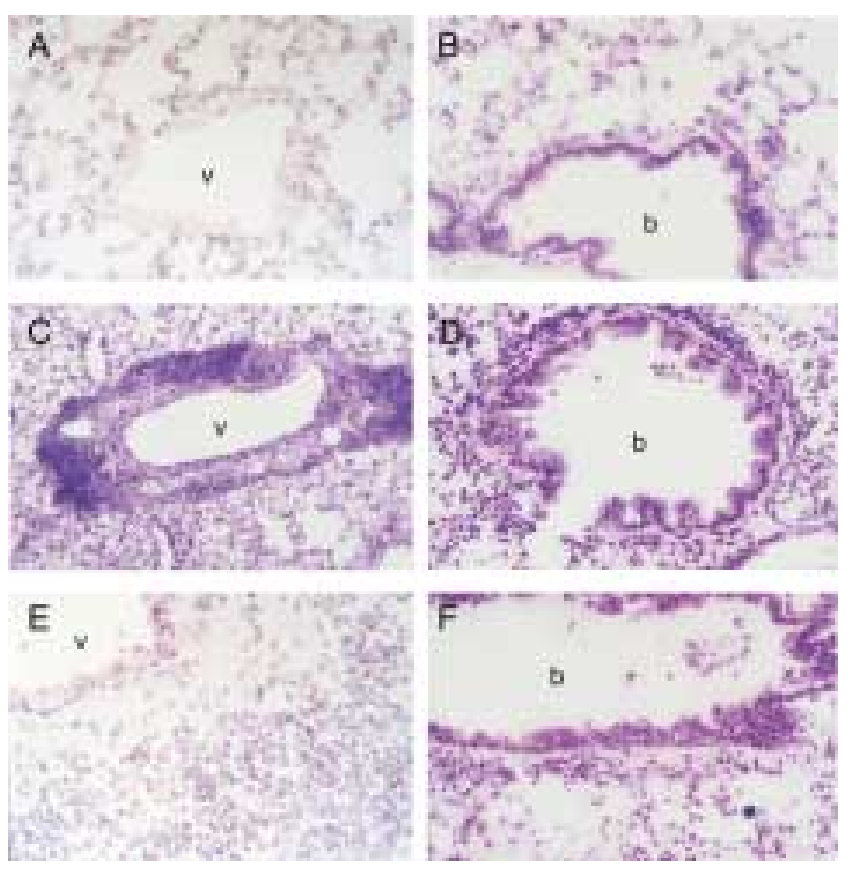

Fig. 5. Lung histology of conducting airways and parenchyma. Sections of formalin-fixed lung tissue from control mice (A and $B$ ), and from mice intranasally challenged with $B$. malayi $\gamma$-GT $(C, D, E$, and F). V, vascular compartment; b, bronchiolus. C and E, Dense perivascular infiltration with leukocytes is shown after the intranasal challenge with $\gamma$-GT. D and C severe peribronchial inflammation. Sections $\mathbf{A}$ and $\mathbf{E}$ were stained with Red Sirius F3B to visualize eosinophils, clearly visible as red spots in panel E. The airways epithelium was enlarged compared to vehicle-sensitized mice, which did not show any histological changes (B). Sections B, $\mathrm{C}, \mathrm{D}$, and $\mathrm{F}$ were stained with hematoxylin. A, B, D $\times 320$; $\mathrm{C} \times 220 \mathrm{E}$, and $\mathrm{F} \times 520$. lar inflammatory lesions. Furthermore, we detected an intense alveolitis characterized by a predominance of neutrophils, mononuclear cells, lymphocytes, and eosinophils detected as nonsegmented, segmented, and bilobed nuclei (Figure 5, C-F). These changes were absent from the lungs of control mice (Figure 5, A and B). The inflammatory cells were grouped into large multifocal cellular infiltrates and located in the mucosa of bronchi, the bronchioli, in the wall of alveoli, and around blood vessels. The bronchial epithelium showed signs of hypertrophy and shedding.

\section{Effect of Intranasal Challenge with Filarial $\gamma$-GT on BAL} Fluid Cytokine Pattern

Considerable evidence supports the notion that IL-4 and IFN- $\gamma$ are key cytokines in the regulation of inflammatory responses in the lung. To determine the type of immune response induced in the airways of mice, BAL fluid was obtained $72 \mathrm{hr}$ after the intranasal antigen challenge with $\gamma$-GT. Figure 6 illustrates IL-4 and IFN- $\gamma$ levels after antigen challenge.
A

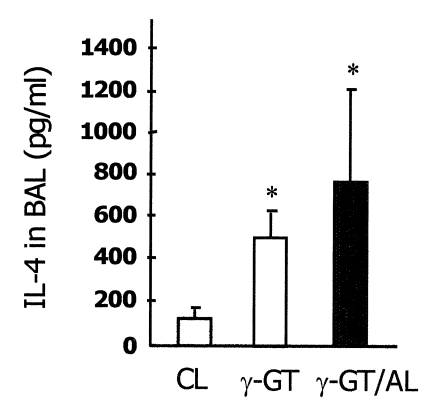

B

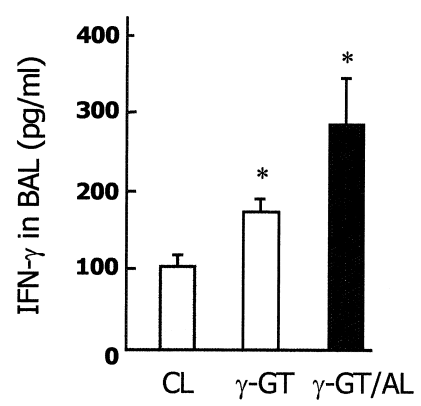

Fig. 6. Levels of IL-4 (A) and IFN- $\gamma$ (B) in the BAL fluid from mice $\gamma$-GT-sensitized and $\gamma$-GT-challenged in the presence or absence of adjuvant $72 \mathrm{hr}$ after intranasal challenge with $\gamma$-GT, $\gamma$-GT/AL or vehicle. Samples were tested in triplicate and data represent the mean \pm SD $(n=4)$. *significant differences between the $\gamma$-GT-immunized mice and the control group (CL). 
Significant elevations of both IL- 4 and IFN- $\gamma$ levels were measured for both cytokines $(p=0.021$ and 0.023 , respectively).

Immunization of Mice with B. malayi $\gamma$-GT Produces Specific Cross-reactive Responses to Endogenous Murine $\gamma$-GT on Airway Epithelial Cells

We had previously identified by molecular cloning a major $B$. malayi allergen in patients with acute TPE as a homolog of human $\gamma-\mathrm{GT}$ with the potential to induce autoreactivity against pulmonary epithelium. Therefore, we determined whether the in vivo immune reactions to the parasite's protein would lead to immune recognition of murine $\gamma$-GT. Indeed, murine affinity purified antibodies to $B$. malayi $\gamma-\mathrm{GT} * * * *$ recognized and bound strongly to the apical surface of airways epithelial cells (Figure 7, C and D). Affinity purified Ab from pre-immune sera showed no interaction with any structure on the airways epithelial cells (Figure 7, A and B). To determine whether the protein on the murine airways epithelial cells recognized by the affinitypurified $A b$ was in fact $\gamma-G T$, we ran native gels from lung extracts and performed in situ staining of $\gamma$-GT enzymatic activity. Immunoblotting of the isolated active murine enzyme using the same affinity purified antibodies from mice immunized against the parasite's $\gamma$-GT revealed a densely stained band of $55 \mathrm{kDa}$ corresponding to the heavy chain (HC) of murine $\gamma$-GT (Figure 8, lane 2). An additional less intensely stained high molecular band of $\sim \mathrm{kDa} 150$, presumably representing a membrane-bound enzyme or alternatively a $\gamma$-GTprecursor, was detected as shown in Figure 8, lane 2. No such bands were detected with serum from control mice, as seen in lane 1. Therefore, these results from the animal model indicate that a breakdown of tolerance had occurred resulting in humoral autoimmunity to murine $\gamma$-GT.
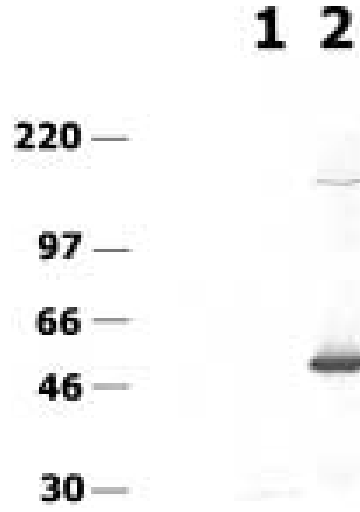

Fig. 8. Autoantibodies against lung murine $\gamma$-GT in sera from mice immunized with $B$. malayi $\gamma$-GT. Western blot analysis of enzymatically-active murine $\gamma$-GT isolated from a nondenaturing gel. Lane 1 shows the immunoblot with control pre-immune serum. Lane 2, immunoblot analysis using affinity purified sera from B. malayi $\gamma$-GT immunized mice demonstrating autoantibodies against the $55 \mathrm{kDa}$ murine heavy chain band, and a weaker $\sim 150 \mathrm{kDa}$ polypeptide representing possible membrane-bound enzyme. No such bands were detected in the controls.

\section{Discussion}

In this study, we have investigated the immune response to filarial $\gamma$-GT in mice and its immunological cross-reactivity with endogenous murine $\gamma$-GT. We have characterized the isotypic pattern of antibodies in Balb/c mice to B. malayi $\gamma$-GT administered with or without alum, as well as the potential role of this parasite antigen in pulmonary inflammation by analyzing cellular and histological changes in the lungs of sensitized mice after intranasal challenge with filarial $\gamma$-GT. The administration of $\gamma$-GT aqueous or alum-precipitated induced high levels of specific IgGs and IgE, transient elevated IgA, and moderate eosinophilia, thus confirming that the murine

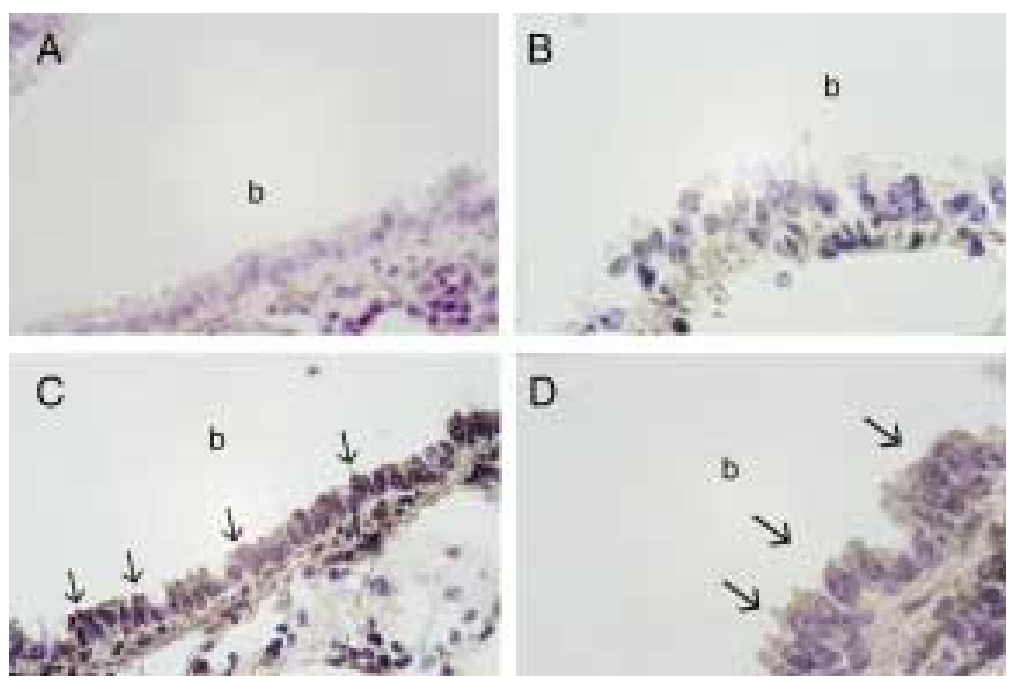

Fig. 7. Lung immunohistochemistry using antibodies from $B$. malayi $\gamma$-GT-immunized mice showing specific autoimmune responses to endogenous murine $\gamma$-GT on airway epithelial cells from the bronchi. Lung sections with bronchi (b) from normal mice were incubated overnight at $37^{\circ} \mathrm{C}$ either with control serum A and B or with affinity-purified anti-B. malayi $\gamma$-GT antibodies $(C$ and $D)$. Panels $C$ and D reveal the presence of autoimmune reactions (black) against the endogenous enzyme detected mainly on the airway epithelium (arrows). These reactions were absent from the sera of control animals ( $\mathrm{A}$ and $\mathrm{B}$ ). Original magnification $\mathrm{A}$ and $\mathrm{C} \times 620 ; \mathrm{B}$ and $\mathrm{D} \times 840$. 
model developed with this mf-derived allergen reflects certain aspects of the human disease. Furthermore, the capacity of $B$. malayi $\gamma$-GT to induce high levels of specific IgE was not dependent on adjuvant (alum) for immunization. Very few recombinant allergens alone have been shown to have the capacity to induce specific IgE antibodies in mice and monkeys (18-20), even those that have may have been contaminated by the presence of LPS in the recombinant protein preparations in some cases.

Immunization with $\gamma$-GT resulted in the production of IFN- $\gamma$ and IL-4, a characteristic pattern of Th0 cells or of a mixed Th1/Th2 response. This was reflected by the in vivo production of IgG2a and IgG3 immunoglobulins, which are up-regulated by IFN- $\gamma$ (21-23), and the production of IgGl and IgE Ab, which are up-regulated by IL-4 (24). Our results confirm findings reported by other investigators showing an in vivo induction of both Th1 and Th2 responses against particulate proteins $(25,26)$. These results are in contrast to the exclusively Thl response resulting from single immunization with $\mathrm{Mf}$ or total microfilarial protein extracts reported previously and confirmed by us $(11,27)$. Interestingly, $B$. malayi $\gamma$-GT-specific IL-4 and $B$. malayi $\gamma$-GT-specific IgE responses were induced much earlier than with immunizations with other filarial antigens or recombinant antigens $(25,27)$. Early induction of antigenspecific IL-4 by microfilarial antigens such as $B$. malayi $\gamma$-GT might contribute to the later development of a Th2-skewed response. High levels of murine $\gamma$-GT-specific IgE antibodies might have also an amplification effect because antigen-specific IgE antibodies enhance in vitro antigen presentation, Tcell proliferation, and specific IgE synthesis (28). A predominance of IgGI and IgE antibodies over the IgG2 response was evident after repeated antigen immunization, as seen on day 65. Comparative analysis of the cytokine and isotype-specific immune responses in the two groups of filarial $\gamma$-GTimmunized animals revealed that the presence of alum as adjuvant only slightly enhanced the level of response but did not alter the cytokine and antibody isotypes response patterns.

Although certain clinical features of TPE suggest involvement of immediate hypersensitivity reaction Type I, involving Ag-specific IgE, other clinical and radiological features of patients with acute TPE reveal a more severe disease state than would result from Type I alone. Therefore, it has been suggested that Type III (antigen-antibody complex) reactions also contribute to the pathogenesis of this syndrome (29). Murine IgGl Abs are able to trigger immediate hypersensitivity reactions independently from IgE (30) and airway hyper-responsiveness (31). There are marked differences in the pathogenic activities of IgG subclasses, e.g., IgG2a autoantibodies, through their capacity to interact with phagocytic Fc $\gamma$ Rs, exhibit the highest pathogenicity in an animal model of autoimmune haemolytic anaemia (32). Dominant
IgG3 immune responses have been observed in patients with bancroftian filariasis (33). Interestingly, a number of autoimmune diseases such as lupus erythematosus cause elevation of IgG3 antibodies, suggesting their involvement in tissue damage through interaction with $\mathrm{Fc} \gamma \mathrm{Rs}$ and through nephritogenic activities of IgG3 antibodies $(34,35)$. Thus, the high levels of $\gamma$-GT-specific IgG1, IgG2a, and IgG3 subclasses detected prior to the intranasal antigen challenge, as well as the membrane-bound location of the target antigen with formation of immune complexes, strongly suggest their participation in the severe pulmonary inflammation in this animal model. Both IgG and IgE immune complexes are potent stimuli for neutrophil activation, leading to tissue damage through the release of reactive oxygen species (28). The elevated levels of murine $\gamma$-GTspecific IgGl, IgA and of $\gamma$-GT-specific IgE induced by this recombinant filarial allergen in the BALB/C model accords with the extraordinarily elevated concentrations of $\gamma$-GT-specific IgGl and IgE concentrations found in patients with acute TPE (manuscript in preparation). This points to a role for these antibodies in the context of Mf-induced pulmonary pathology. In addition, recombinant $B$. malayi $\gamma$-GT was also able to induce IgA antibodies in the mouse model, suggesting not only enhanced humoral immunity but also up-regulation of mucosal immunity.

Interestingly, inhibition of $\gamma$-GT activity present on human macrophages has induced transforming growth factor- $\beta 1$ (36), a key cytokine involved in pathological processes in the lung, such as the induction of fibrosis (37). The presence of TGF- $\beta 1$ in the context of a self-antigen (islet antigen) is associated with a deviation of the immune response of antigen-presenting cells toward a Th2 phenotype (38). We are currently investigating whether TGF- $\beta 1$ might be released either after intranasal challenge with this filarial allergen or from cultured human lung epithelial cells to clarify downstream mechanisms of pathology mediated by autoimmune $\gamma$-GT antibodies.

The lungs from both groups of sensitized and intranasally challenged mice showed massive pulmonary inflammation with peribronchial, perivascular, and alveolar cellular infiltration. Early animal studies on the pulmonary pathology associated with microfilarial clearance in dogs infected with the filarial parasite Dirofilaria immitis demonstrated the development of pulmonary fibrosis in chronic occult filarial infections (39). Similarly, investigations on the pulmonary pathology in jirds infected with $B$. malayi and $B$. pahangi revealed the development of endarteritis and interstitial inflammation, with thickening of intima and subintimal layers (40). Whether or not pulmonary fibrosis might develop from prolonged immunization with this nematode allergen remains to be elucidated.

The appearance of both antigen-induced IFN- $\gamma$ and IL-4 in the BAL after the immunization and 
intranasal challenge might contribute to airway inflammation. Adoptive transfer studies have shown that OVA-specific Thl cells caused severe pulmonary inflammation and did not reduce the inflammatory effects of Th2 cells, demonstrating that both types of T-helper cells contribute to the pathology in this model of pulmonary inflammation (41). Furthermore, very recently it was shown that cooperation between the different Th1- and Th2-helper cells, rather than mutual exclusion, promotes airway inflammation (42). Virally transformed $\mathrm{T}$ cells of the Th0, Th1, and Th2 types have been obtained from patients with TPE; however, the antigen specificity of these $\mathrm{T}$ cells has not yet been established (43). Further studies using B. malayi $\gamma$-GT-specific Th-cell clones are required to elucidate their specific contribution to the observed airway inflammation.

Molecular mimicry between infectious agents and self components is postulated as a mechanism to account for the antigen and organ specificity of autoimmune responses in infectious diseases $(44,45)$. Although the overall sequence homology between the heavy chains of the filarial and mouse $\gamma$-GTs is only $37 \%$, a detailed comparison of selected amino acid areas revealed homologies of $>80 \%$. Future analysis using peptides spanning selected areas of the filarial $\gamma$-GT should provide insight into the mechanisms of the breakdown of tolerance.

The present study provides support for molecular mimicry between a major parasite allergen and a self-protein present on the airway epithelium as a mechanism of induction of autoantibodies. Affinity purified antibodies or sera from immunized animals reacted mainly with the apical surface of airways epithelium where $\gamma$-GT has previously been shown to be located (46-48). The apparent molecular mass of the polypeptide detected by the autoantibodies corresponds to the apparent molecular mass of the murine $\gamma$-GT's HC reported for other mice strains (49). In addition, we have evidence that immune responses induced by the parasite's $\gamma$-GT have a profound impact on the intracellular glutathione homeostasis of the lungs of immunized mice and therefore on their capacity to withstand oxidative stress (manuscript in preparation).

The findings presented here indicate that autoimmunity induced by a microfilarial antigen with homology to a host enzyme on the pulmonary epithelium may contribute to the pathogenesis of the TPE syndrome. Future studies using recently generated murine B cell hybridomas secreting $B$. malayi $\gamma$-GT-specific IgE and IgG subclass antibodies in a transfer model will clarify the role of subclassspecific Abs in the observed pathology as well as the definition of pathogenic B epitopes on $\gamma$-GT. In addition, the membrane localization of $\gamma$-GT on airways epithelial cells, and the availability of $\gamma$ GT-specific IgE mAb may allow the establishment of a novel molecular model to study allergen-IgEFceRs interactions. A better understanding of the pathogenesis of the different clinical manifestations of lymphatic filariasis is crucial for the development of the different strategies aiming to avoid and/or minimize the devastating effects of this disease.

\section{Acknowledgments}

We thank the Filariasis Repository from the NIAID/NIH, Bethesda, MD, USA for providing $B$. malayi microfilariae used in the present study, and Annie Higgs for critical reading of the manuscript. This work was supported by a research grant (052842) from the Wellcome Trust.

\section{References}

1. Ottesen EA, Neva FA, Paranjape RS, Tripathy SP, Thiruvengadam KV, Beaven MA. (1979) Specific allergic sensitisation to filarial antigens in tropical eosinophilia syndrome. Lancet 1 : 1158-1161.

2. Neva FA, Ottesen EA. (1978) Tropical (filarial) eosinophilia. N. Engl. J. Med. 298: 1129-1131.

3. Spry CJ, Kumaraswami V. (1982) Tropical eosinophilia. Semin. Hematol. 19: 107-115.

4. Udwadia F. (1967) Tropical eosinophilia: a correlation of clinical histopathological and lung function studies. Dis. Chest. 52: 531-538.

5. Nutman TB, Vijayan VK, Pinkston P, et al. (1989) Tropical pulmonary eosinophilia: analysis of antifilarial antibody localized to the lung. J. Infect. Dis. 160: 1042-1050.

6. Lobos E, Ondo A, Ottesen EA, Nutman TB. (1992) Biochemical and immunologic characterization of a major IgE-inducing filarial antigen of Brugia malayi and implications for the pathogenesis of tropical pulmonary eosinophilia. J. Immunol. 149: 3029-3034.

7. Lobos E, Zahn R, Weiss N, Nutman TB. (1996) A major allergen of lymphatic filarial nematodes is a parasite homolog of the gamma-glutamyl transpeptidase. Mol. Med. 2: 712724.

8. Lieberman MW, Barrios R, Carter BZ, et al. (1995) Gammaglutamyl transpeptidase. What does the organization and expression of a multipromoter gene tell us about its functions? Am. J. Pathol. 147: 1175-1185.

9. Egwang TG, Kazura JW. (1990) The BALB/c mouse as a model for immunological studies of microfilariae-induced pulmonary eosinophilia. Am. J. Trop. Med. Hyg. 43: 61-66.

10. Pearlman E, Kroeze WK, Hazlett FE, et al. (1993) Brugia malayi: acquired resistance to microfilariae in BALB/c mice correlates with local Th2 responses. Exp. Parasitol. 76: 200-208.

11. Pearlman E, Hazlett FE, Jr., Boom WH, Kazura JW. Induction of murine T-helper-cell responses to the filarial nematode Brugia malayi. (1993) Infect. Immun. 61: 1105-1112.

12. Modolell M, Corraliza IM, Link F, Soler G, Eichmann K. (1995) Reciprocal regulation of the nitric oxide synthase/arginase balance in mouse bone marrow-derived macrophages by THI and TH2 cytokines. Eur. J. Immunol. 25: 1101-1104.

13. Coyle AJ, Wagner K, Bertrand C, Tsuyuki S, Bews J, Heusser C. (1996) Central role of immunoglobulin (Ig) $\mathrm{E}$ in the induction of lung eosinophil infiltration and T helper 2 cell cytokine production: inhibition by a non-anaphylactogenic anti-IgE antibody. J. Exp. Med. 183: 1303-1310.

14. Bogomeletz W. (1980) Avantages de la coloration par le rouge sirius de l'amyloide et des eosinophiles. Arch. Anat. Cytol. Path. 28: 252-253.

15. Ricken AM, Spanel-Borowski K, Saxer M, Huber PR. (1995) Cytokeratin expression in bovine corpora lutea. Histochem. Cell. Biol. 103: 345-354.

16. Glass GA, Bluvshtein E, Gur Y, Sfez S, Simovich V, Stark AA. (1994) Detection of conformational changes in rat kidney gamma-glutamyl transpeptidase by an antibody against a 
synthetic peptide belonging to part of the reactive centre of the enzyme. Biochem. Mol. Biol. Int. 33: 505-513.

17. Kugelman A, Choy HA, Liu R, Shi MM, Gozal E, Forman HJ. (1994) Gamma-glutamyl transpeptidase is increased by oxidative stress in rat alveolar L2 epithelial cells. Am. J. Respir. Cell. Mol. Biol. 11: 586-592.

18. Vrtala S, Mayer P, Ferreira F et al. (1996) Induction of IgE antibodies in mice and rhesus monkeys with recombinant birch pollen allergens: different allergenicity of Bet $\mathrm{v} 1$ and Bet $\mathrm{v} 2$. J. Allergy. Clin. Immunol. 98: 913-921.

19. Wang H, Mao X, Simons FE, Peng Z. (1999) Induction of IgE responses using a recombinant mosquito salivary allergen rAed a 2 without adjuvant in mice. Int. Arch. Allergy Immunol. 120: $135-140$.

20. Ghosh K, Hotez PJ. (1999) Antibody-dependent reductions in mouse hookworm burden after vaccination with Ancylostoma caninum secreted protein 1. J. Infect. Dis. 180: 16741681.

21. Snapper CM, Paul WE. (1987) Interferon-gamma and B cell stimulatory factor-1 reciprocally regulate Ig isotype production. Science 236: 944-947.

22. Snapper CM, Peschel C, Paul WE. (1988) IFN-gamma stimulates IgG2a secretion by murine B cells stimulated with bacterial lipopolysaccharide. J. Immunol. 140: 2121-2127.

23. Snapper CM, McIntyre TM, Mandler R, et al. (1992) Induction of IgG3 secretion by interferon gamma: a model for $T$ cell-independent class switching in response to $T$ cellindependent type 2 antigens. J. Exp. Med. 175: 1367-1371.

24. Snapper CM, Finkelman FD, Paul WE. (1988) Regulation of IgGl and IgE production by interleukin 4. Immuno.l Rev. 102: 51-75.

25. Comoy EE, Capron A, Thyphronitis G. (1997) In vivo induction of type 1 and 2 immune responses against protein antigens. Int. Immunol. 9: 523-531.

26. Sedlik C, Deriaud E, Leclerc C. (1997) Lack of Th1 or Th2 polarization of $\mathrm{CD} 4+\mathrm{T}$ cell response induced by particulate antigen targeted to phagocytic cells. Int. Immunol. 9: 91103.

27. Lawrence RA, Allen JE, Osborne J, Maizels RM. (1994) Adult and microfilarial stages of the filarial parasite Brugia malayi stimulate contrasting cytokine and Ig isotype responses in BALB/c mice. J. Immunol. 153: 1216-1224.

28. Zuberi RI, Apgar JR, Chen SS, Liu FT. (2000) Role for IgE in airway secretions: IgE immune complexes are more potent inducers than antigen alone of airway inflammation in a murine model. J. Immunol. 164: 2667-2673.

29. Ottesen EA. (1976) Eosinophilia and the Lung. In: Kirckpatrick $\mathrm{CH}$, Reynolds HY, eds. Immunologic and Infectious Reactions in the Lung. New York: Dekker M: 289-332.

30. Oettgen HC, Martin TR, Wynshaw-Boris A, Deng C, Drazen JM, Leder P. (1994) Active anaphylaxis in IgE-deficient mice. Nature 370: 367-370.

31. Oshiba A, Hamelmann E, Takeda K, et al. (1996) Passive transfer of immediate hypersensitivity and airway hyperresponsiveness by allergen-specific immunoglobulin (Ig) E and IgG1 in mice. J. Clin. Invest. 97: 1398-1408.

32. Fossati-Jimack L, Ioan-Facsinay A, Reininger L, et al. (2000) Markedly different pathogenicity of four immunoglobulin $G$ isotype-switch variants of an antierythrocyte autoantibody is based on their capacity to interact in vivo with the low-affinity Fcgamma receptor III. J. Exp. Med. 191: 1293-1302.

33. Kazura JW, Hazlett FE, Jr., Pearlman E, et al. (1992) Antigenicity of a protective recombinant filarial protein in human bancroftian filariasis. J. Infect. Dis. 166: 1453-1457.

34. Maddison PJ. Autoantibodies in SLE. (1999) Disease associations. Adv. Exp. Med. Biol. 455: 141-145.

35. Reininger L, Berney $T$, Shibata T, Spertini F, Merino R, Izui S. (1990) Cryoglobulinemia induced by a murine IgG3 rheumatoid factor: skin vasculitis and glomerulonephritis arise from distinct pathogenic mechanisms. Proc. Natl. Acad. Sci. USA 87: 10038-10042.

36. Bauvois B, Laouar A, Rouillard D, Wietzerbin J. (1995) Inhibition of gamma-glutamyl transpeptidase activity at the surface of human myeloid cells is correlated with macrophage maturation and transforming growth factor beta production. Cell. Growth. Differ. 6: 1163-1170.

37. Branton MH, Kopp JB. (1999) TGF-beta and fibrosis. Microbes. Infect. 1: 1349-1365.

38. King C, Davies J, Mueller R, et al. (1998) TGF-betal alters APC preference, polarizing islet antigen responses toward a Th2 phenotype. Immunity 8: 601-613.

39. Castleman WL, Wong MM. (1982) Light and electron microscopic pulmonary lesions associated with retained microfilariae in canine occult dirofilariasis. Vet. Pathol. 19: 355-364.

40. Vincent AL, Frommes SP, Ash LR. (1976) Brugia malayi, Brugia pahangi, and Brugia patei: pulmonary pathology in jirds, Meriones unguiculatus. Exp. Parasitol. 40: 330-354.

41. Hansen G, Berry G, DeKruyff RH, Umetsu DT. (1999) Allergenspecific Thl cells fail to counterbalance Th2 cell-induced airway hyperreactivity but cause severe airway inflammation. J. Clin. Invest. 103: 175-183.

42. Randolph DA, Stephens R, Carruthers CJ, Chaplin DD. (1999) Cooperation between Thl and Th2 cells in a murine model of eosinophilic airway inflammation. J. Clin. Invest. 104: 1021-1029.

43. Nutman TB. (1992) T-cell regulation of immediate hypersensitivity: lessons from helminth parasites and allergic diseases. In: Moqbel R, ed. Allergy and Immunity to Helminths. London and Washington, DC: Taylor and Francis: 187-204.

44. Ehrenstein M, Isenberg D. (1991) Autoimmunity associated with infection: leprosy, acute rheumatic fever and Lyme disease. Curr. Opin. Immunol. 3: 930-935.

45. Rose NR. (1998) The role of infection in the pathogenesis of autoimmune disease. Semin. Immunol. 10: 5-13.

46. Ingbar DH, Hepler $K$, Dowin $R$, et al. (1995). GammaGlutamyl transpeptidase is a polarized alveolar epithelial membrane protein. Am. J. Physiol. 269: L261-L271.

47. Oakes SM, Takahashi Y, Williams MC, Joyce-Brady M. (1997) Ontogeny of gamma-glutamyltransferase in the rat lung. Am. J. Physiol. 272: L739-L744.

48. Hagen TM, Brown LA, Jones DP. (1986) Protection against paraquat-induced injury by exogenous GSH in pulmonary alveolar type II cells. Biochem. Pharmacol. 35: 4537-4542.

49. Harding CO, Williams P, Wagner E, et al. (1997) Mice with genetic gamma-glutamyl transpeptidase deficiency exhibit glutathionuria, severe growth failure, reduced life spans, and infertility. J. Biol. Chem. 272: 12560-12567. 